\title{
Interleukin-1 $\beta$ activates focal adhesion kinase and Src to induce matrix metalloproteinase-9 production and invasion of MCF-7 breast cancer cells
}

\author{
NAING NAING MON, TAKESHI SENGA and SATOKO ITO \\ Division of Cancer Biology, Nagoya University Graduate School of Medicine, Showa-ku, Nagoya 466-8550, Japan
}

Received September 28, 2015; Accepted October 14, 2016

DOI: $10.3892 / \mathrm{ol} .2016 .5521$

\begin{abstract}
Interleukin-1 $\beta$ (IL-1b) is a pleiotropic cytokine that is important in tumor progression and invasion. Matrix metalloproteinase-9 (MMP-9), which is a secreted matrix-degrading enzyme, is one of the key regulators of tumor invasion and metastasis. The current report indicated that IL-1b promotes MMP-9 production and cell invasion in non-metastatic MCF-7 breast cancer cells. IL-1b activated focal adhesion kinase $(\mathrm{FAK})$ and proto-oncogene tyrosine-protein kinase Src (Src). Moreover, inhibiting the Src/FAK pathway reduced the IL-1b-induced production of MMP-9 and cell invasion. To investigate the functional role of FAK in MMP-9 production cell lines expressing mutant FAK in FAK knock-out mouse fibroblasts were generated. In wild-type FAK-expressing cells, MMP-9 production was induced by IL-1b stimulation. By contrast, IL-1b-induced MMP-9 production was abrogated in FAK knock-out, FAK Y397F, FAK Y925F, and kinase dead mutant-expressing cells. Therefore the results of the current study indicate that FAK and Src kinases are activated by IL-1b and play a critical role in MMP-9 production and tumor cell invasion.
\end{abstract}

\section{Introduction}

A number of epidemiological and clinical studies have presented evidence that chronic inflammation caused by microbial infection, as well as chemical irritants, significantly increases cancer risk $(1,2)$. Obesity and post-partum involution are chronic inflammatory states in mammary glands, and are associated with increased breast cancer risk (3). Furthermore, non-steroidal anti-inflammatory drugs (NSAID), which are nonselective Prostaglandin $\mathrm{G} / \mathrm{H}$ synthase 2, or cyclooxygenase-2 (COX-2) inhibitors, significantly reduce the risk of mammary carcinogenesis, recurrence, and motility of breast

Correspondence to: Dr Satoko Ito, Division of Cancer Biology, Nagoya University Graduate School of Medicine, 65 Tsurumai-cho, Showa-ku, Nagoya 466-8550, Japan

E-mail: s-ito@med.nagoya-u.ac.jp

Key words: FAK, Src, MMP-9, IL-1 $\beta$, invasion, breast cancer cell cancer (4). In the tumor microenvironment, various inflammatory cells, such as tumor-associated macrophages, are recruited to form a pro-tumor inflammatory environment. Inflammatory cells produce a variety of mediators, including growth factors, chemokines and cytokines, which induce tumor growth, invasion, and angiogenesis $(5,6)$.

Proinflammatory cytokines are a major determinant for the invasiveness of tumor cells. Among these cytokines, interleukin-1 $\beta$ (IL-1 $\beta$ ) is abundant in tumor tissues and stimulates tumor growth, invasion, carcinogenesis and host-tumor association (7). IL-1 $\beta$-knockout mice are resistant to the development of chemically induced tumors (8), and exhibit suppressed tumor invasion and angiogenesis $(9,10)$. On the other hand, stomach-specific IL-1 $\beta$ overexpression induces gastric inflammation and cancer (11). These results identify IL-1 $\beta$ as one of the essential components mediating inflammation-associated tumor progression. IL-1 $\beta$ stimulation of tumor cells activates multiple signaling pathways involving protein kinase $\mathrm{B}$, mitogen activated protein (MAP) kinase, and nuclear factor- $\kappa B$ (12). Activation of these signaling molecules is required for IL-1 $\beta$-mediated production of matrix metalloproteinase (MMP)-9, a matrix degrading enzyme that is regarded as a critical regulator in IL-1 $\beta$-induced tumor invasion (13-15). A number of studies have investigated the association between MMP expression and the prognosis of breast cancer patients. Most recently, a meta-analysis by Ren et al (16) reported that MMP-9 overexpression in serum was associated with poor patient prognosis in breast cancer.

Proto-oncogene tyrosine-protein kinase $\mathrm{Src}(\mathrm{Src})$ is a non-receptor tyrosine kinase that is comprised of $\mathrm{SH} 3, \mathrm{SH} 2$, and kinase domains. Extracellular stimuli including cytokines, growth factors and integrin engagement, activate Src, which in turn, phosphorylates various target proteins to regulate cell proliferation, differentiation, and migration $(17,18)$. Among these target proteins, focal adhesion kinase 1 (FAK) is essential for the regulation of signal transduction, cell adhesion and migration carried out by Src (19). FAK is composed of an N-terminal FERM domain, a central kinase domain, and a C-terminal focal adhesion targeting domain. Additionally, FAK localizes to the site of cell-extracellular matrix contact (20). There are six major tyrosine phosphorylation sites in FAK, and two of them, the Tyr397 and Tyr925 sites, are important for FAK-dependent signaling (21). Src interacts with the phosphorylated Tyr397 of FAK and phosphorylates 
Tyr925, which in turn associates with signaling molecules such as growth factor receptor-bound protein 2 (Grb2) to induce activation of the Ras-dependent/MAP kinase pathway $(19,22)$.

It has previously been reported by this group that the enhancement of cell invasion caused by nitric oxide stimulation is mediated by Src and FAK kinase activation in MCF-7 breast cancer cells (23). To expand on these findings, the current study aimed to examine the role Src and FAK serve in the IL-1 $\beta$-mediated cell invasion of MCF-7 cells, and identify whether Src and FAK kinase are involved in MMP-9 production and cell invasion.

\section{Materials and methods}

Antibodies, cytokines and chemicals. Recombinant murine IL-1b and recombinant human IL-1b were purchased from PeproTech EC (London, UK). The PP2 kinase inhibitor (PP2) was purchased from EMD Millipore (Billerica, MA, USA). Anti-FAK (cat. no. sc-154; 1:1,000), anti-phospho-Erk (cat. no. sc-7383; 1:1,000) and anti-Erk2 (cat. no. sc-558; 1:1,000) antibodies were all obtained from Santa Cruz Biotechnology (Dallas, TX, USA); anti-phosphotyrosine antibody (pTyr20; cat. no. 610012; 1:1,000) was purchased from BD Transduction Laboratories $^{\mathrm{TM}}$ (BD Biosciences, Franklin Lakes, NJ USA); anti-phospho-FAK antibody (pTyr397; cat. no. 44624G; 1:500) was purchased from Invitrogen (Thermo Fisher Scientific, Inc., Waltham, MA, USA); and anti-phospho-Src (pTyr416; cat. no. 6943; 1:1,000) and anti-phospho-FAK (pTyr925; cat. no. $3284 ; 1: 1,000)$ antibodies were both obtained from Cell Signaling Technology (Danvers, MA, USA).

Cell culture, plasmid construction, and transfection. The human breast cancer cell line, MCF-7, was obtained from the Japanese Collection of Research Bioresources Cell Bank (Osaka, Japan), and cultured in Dulbecco's modified Eagle's medium (DMEM) supplemented with $10 \%$ fetal bovine serum (Biowest Europe, Nuaillé, France) and $5 \mathrm{mg} / \mathrm{ml}$ human insulin (Sigma-Aldrich; Merck Millipore, Darmstadt, Germany). Subsequently, knockout of FAK was performed to establish homozygous null FAK knockout fibroblast cells, following a previously documented procedure by Ilić et al (24). To establish FAK-wild-type (FAK-Wt), FAK-kinase dead (KD), FAK-Y397F, and FAK-Y925F cells, wild-type and mutant FAKs were cloned into a pBabepuro vector and transfected into FAK-Ko cells.

Assay of gelatin-degrading MMPs by zymography. The activity of MMPs in the conditioned media was assayed by zymography as described previously (21). Briefly, cells were incubated in serum-free medium for $6 \mathrm{~h}$ followed by stimulation with or without IL-1b for $16 \mathrm{~h}$. Conditioned media were collected, clarified by centrifugation, and subjected to electrophoresis with sodium dodecyl sulphate-polyacrylamide gels copolymerized with gelatin. Gels were washed and incubated with reaction buffer $\left(50 \mathrm{mM}\right.$ Tris- $\mathrm{HCl}, \mathrm{pH} 7.4,0.02 \% \mathrm{NaN}_{3}$, $10 \mathrm{mM} \mathrm{CaCl}_{2}$ ) for $16 \mathrm{~h}$ at $37^{\circ} \mathrm{C}$, stained with Coomassie brilliant blue, and subsequently destained.

FAK siRNA and transfection. The sequence of FAK siRNA is 5'-CCACCUGGGCCAGUAUUAUTT-3, and the sequence for luciferase siRNA is 5'-CUUACGCUGAGUACUUCGATT-3'. Cells were transfected with $20 \mathrm{nM}$ of each siRNA using Lipofectamine $^{\mathrm{TM}}$ RNAi/MAX (Invitrogen; Thermo Fisher Scientific, Inc.) according to the manufacturers' protocol.

Invasion assay. MCF-7 cells were assayed for their invasiveness by a modified Boyden chamber method as described previously (21). Cells were serum-starved for $6 \mathrm{~h}$ and pretreated with varying concentrations of IL- $1 \mathrm{~b}(0,0.5,1,3,5,10 \mathrm{nM})$ for $12 \mathrm{~h}$. Cells were subsequently resuspended in serum-free DMEM and seeded onto Matrigel-coated filters with or without IL-1b. Following incubation for $7 \mathrm{~h}$, cells that had invaded the lower surface of the filter were fixed, stained, and quantified by counting three randomly selected fields under the microscope. The mean \pm standard deviation (SD) of three independent experiments was calculated. To examine cell invasion in the absence of FAK expression, cells were incubated with the indicated siRNAs for $30 \mathrm{~h}$, serum-starved for $6 \mathrm{~h}$ and treated with IL-1b for $12 \mathrm{~h}$ before undergoing an invasion assay. To investigate cell invasion with PP2, cells were serum-starved for $6 \mathrm{~h}$, treated with $10 \mu \mathrm{M}$ PP2 for $1 \mathrm{~h}$, stimulated with IL- $1 \mathrm{~b}$ for $12 \mathrm{~h}$ and subjected to an invasion assay.

Statistical analysis. Values are expressed as the mean \pm SD of three independent experiments. Comparisons between groups were performed using an unpaired Student's t-test. Statistical analysis was performed using GraphPad Prism software (version 7.0; GraphPad Software, Inc., La Jolla, CA, USA). $\mathrm{P}<0.05$ were considered to indicate a statistically significant difference.

\section{Results}

$I L-1 b$ induces invasion and MMP-9 production in MCF-7 cells. The effect of IL-1b stimulation on the invasiveness of non-metastatic MCF-7 human breast cancer cells in vitro was examined. The level of invasion by MCF-7 cells was low in the absence of IL-1b stimulation. However, increasing the concentration of IL-1b increased the invasiveness of MCF-7 cells in a dose-dependent manner (Fig. 1A). MMPs are matrix-degrading enzymes essential for tumor cell invasion. Previous studies have demonstrated that IL-1b activates MMP-9 secretion (14). Therefore, the production of MMP-9 in MCF-7 cells following IL-1b stimulation was examined. MCF-7 cells were serum-starved, treated with the indicated concentrations of human IL-1b for $16 \mathrm{~h}$ prior to gelatin zymography. Although MMP-9 production was undetectable in MCF-7 cells in the absence of IL-1b, IL-1b stimulation increased MMP-9 expression in a dose-dependent manner (Fig. 1B). By contrast, the production of MMP-2 was limited even at higher doses of IL-1b (Fig. 1B).

FAK and Src are activated by IL-1b stimulation in MCF-7 cells. It was previously demonstrated by this group that FAK activation is responsible for the increased production of MMP-9 by fibronectin and tumor necrosis factor $\alpha(25,26)$. Thus, the current study investigated whether FAK was activated by IL-1b stimulation. To measure tyrosine phosphorylation of FAK, MCF-7 cells were serum-starved, treated with $3 \mathrm{nM}$ IL-1b, and lysed to immunoprecipitate FAK, and 

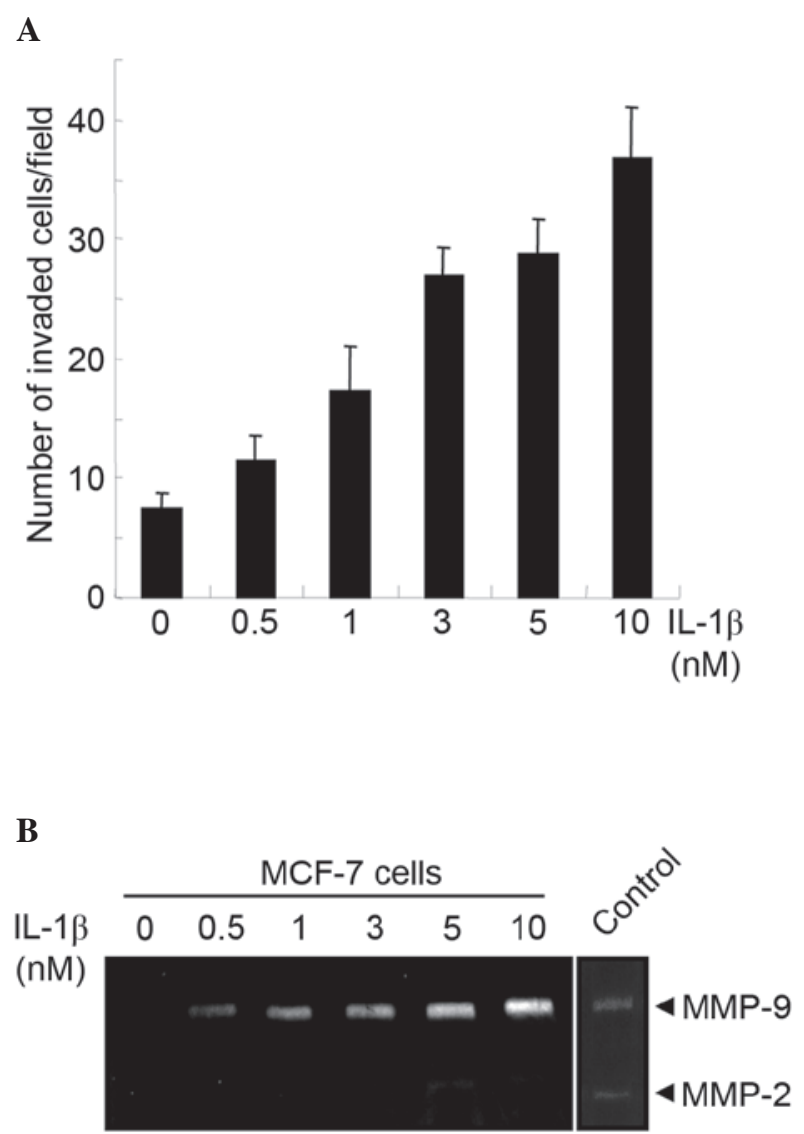

Figure 1. IL-1 $\beta$ induces MMP-9 production and promotes MCF-7 cell invasion. (A) MCF-7 cells were serum-starved for $6 \mathrm{~h}$ and pretreated with the indicated concentrations of human IL-1 $\beta$ for $12 \mathrm{~h}$. Cells were resuspended in serum-free DMEM and seeded onto a Matrigel-coated Boyden chamber with or without IL-1 $\beta$. Following incubation for $7 \mathrm{~h}, \mathrm{MCF}-7$ cells that invaded the lower surface of the filter were fixed, stained and quantified by counting three randomly selected fields under the microscope. Data represent the mean $\pm \mathrm{SD}$ of three independent experiments. (B) MCF-7 cells were serum-starved for $6 \mathrm{~h}$ and incubated with the indicated concentrations of human IL-1 $\beta$ for $16 \mathrm{~h}$ Conditioned media were collected and subjected to gelatin zymography.

western blotting was subsequently carried out. Treatment of MCF-7 cells with IL-1b increased FAK tyrosine phosphorylation in a time-dependent manner (Fig. 2A). Furthermore, the phosphorylation of Tyr397 and Tyr925 in FAK was evaluated. Tyr397 is auto-phosphorylated when the kinase is activated, and Tyr925, which functions as a binding site for Grb2 to activate the Ras/ERK pathway, is phosphorylated by Src. The phosphorylation of these tyrosine residues was induced by IL-1b stimulation (Fig. 2A). Src regulates the phosphorylation of Tyr925, therefore its activation following IL-1b stimulation was assessed. The phosphorylation of Tyr416, which regulates the catalytic activity of Src, was increased by IL-1b stimulation (Fig. 2B).

FAK and Src are required for $I L-1 b$-induced MMP-9 production and invasion in MCF-7 cells. The effect of silencing FAK on the production of MMP-9 in MCF-7 cells was investigated. MCF-7 cells were transfected with FAK siRNA, and the production of MMP-9 after IL-1b treatment was examined by zymography. Knockdown of FAK expression by siRNA reduced the production of MMP-9 by IL-1b (Fig. 3A). The
A

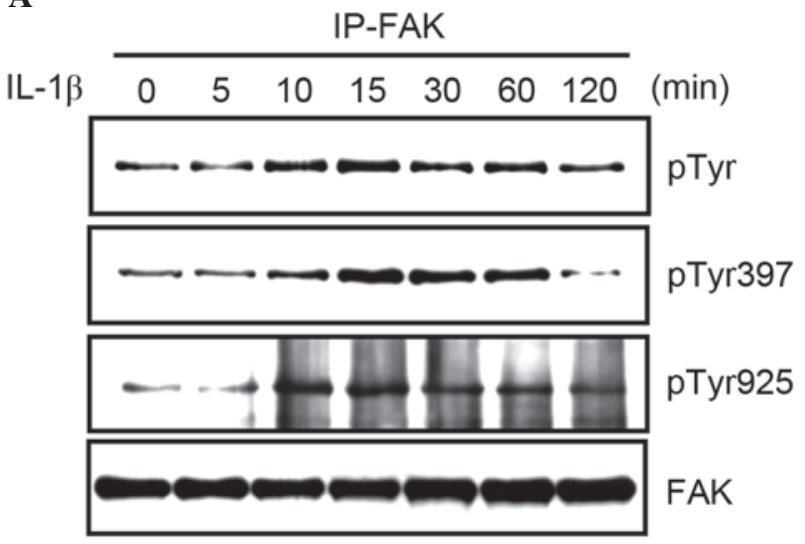

B

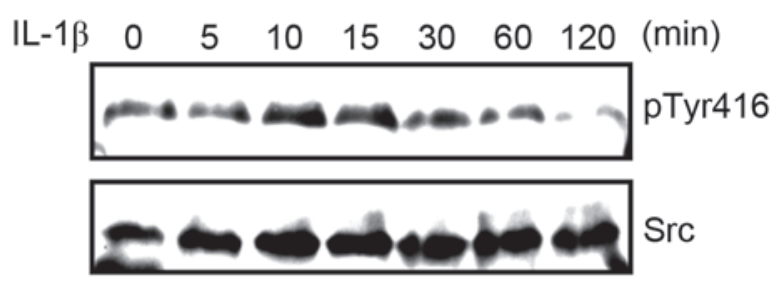

Figure 2. IL-1 $\beta$ activates FAK and Src in MCF-7 cells. (A) MCF-7 cells were serum-starved and stimulated with $3 \mathrm{nM}$ IL-1 $\beta$. FAK was immunoprecipitated at the indicated time points and tyrosine phosphorylation was examined by using an anti-phospho-tyrosine antibody. Phospho-FAK-specific antibodies were used to determine the phosphorylation of Tyr397 and Tyr925. FAK expression acted as a reference (B) MCF-7 cells were serum-starved and stimulated with $3 \mathrm{nM}$ IL-1 $\beta$ for the indicated times, and the phosphorylation of Tyr416 was determined by a phospho-Src-specific antibody. Src expression acted as a reference.

role of FAK in the IL-1b-induced invasion of MCF-7 cells was then examined using a modified Boyden chamber. MCF-7 cells were transfected with either a luciferase or FAK siRNA, and after $30 \mathrm{~h}$, they were starved and stimulated with IL-1b. Cells were then loaded onto the upper chamber and incubated with or without IL-1b. Following $7 \mathrm{~h}$, cells that had invaded the lower surface of the chamber were fixed, stained and quantified by counting. The results demonstrated that FAK siRNA significantly suppressed cell invasion (Fig. 3B).

To determine whether Src is required for IL-1b-mediated production of MMP-9, the Src inhibitor PP2 was used. MCF-7 cells were stimulated with IL-1b with or without PP2, MMP-9 production was examined, and it was observed that PP2 treatment markedly suppressed MMP-9 production (Fig. 3C).

A previous study had demonstrated that the activation of extracellular signal-related kinases (Erk) was required for MMP-9 production (12). The current study demonstrated that stimulation of MCF-7 cells with IL-1b led activated Erk, which in turn was inhibited by PP2 treatment. In addition, the combined treatment of PP2 and FAK siRNA reduced the phosphorylation of Erk, which is mediated by IL-1b (Fig. 3C). This indicates that the Src/FAK pathway is crucial for the activation of Erk by IL-1b, and production of MMP-9.

The requirement of Src for IL-1b-induced invasion of MCF-7 cells was examined using a modified Boyden chamber. 


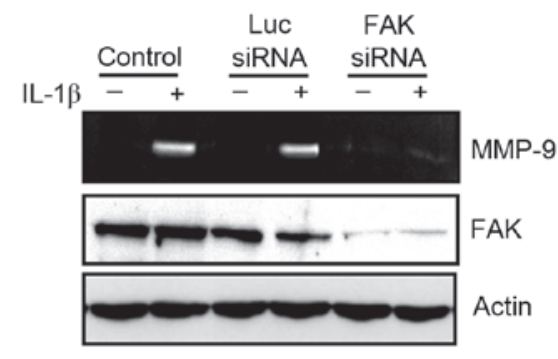

B

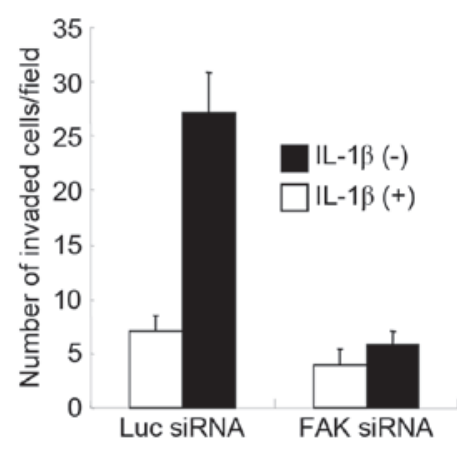

C

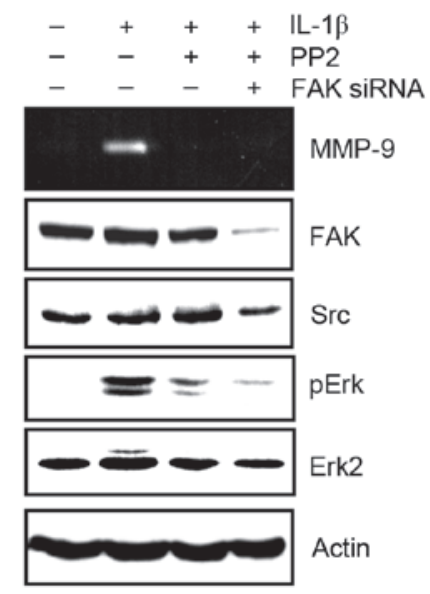

D

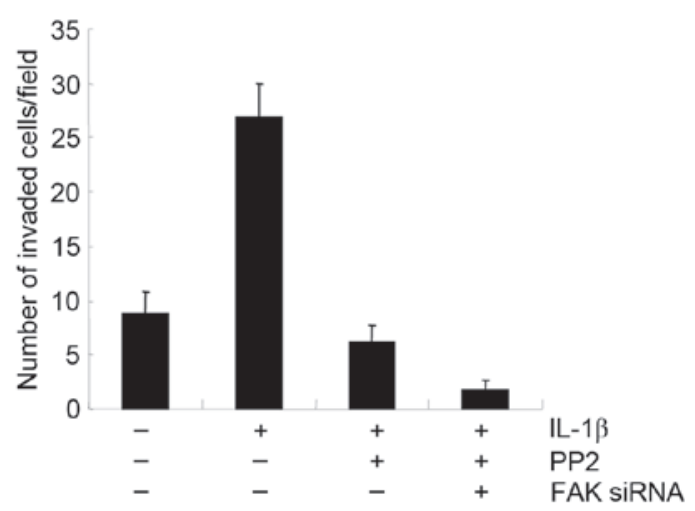

Figure 3. FAK and Src are required for IL-1 $\beta$-induced MMP-9 production and invasion in MCF-7 cells. (A) MCF-7 cells were transfected with either luciferase or FAK siRNA. $30 \mathrm{~h}$ following transfection, cells were serum-starved for $6 \mathrm{~h}$ and incubated with or without $3 \mathrm{nM} 1 \mathrm{~L}-1 \beta$ for $16 \mathrm{~h}$. Conditioned media were collected and subjected to zymography (upper panel). Expression of FAK was determined by western blot analysis (lower panel). Actin expression was used as a reference. (B) Cells were transfected with either luciferase or FAK siRNA and stimulated with IL-1 1 . Cell invasion was determined by using a modified Boyden chamber. Data represent the mean \pm SD from three independent experiments. (C) Following treatment with either luciferase or FAK siRNA for $30 \mathrm{~h}$, cells were serum-starved for $6 \mathrm{~h}$, stimulated by $3 \mathrm{nM} \mathrm{IL-1 \beta}$ for $16 \mathrm{~h}$, and were treated with or without PP 2 for $1 \mathrm{~h}$. Conditioned media were collected and subjected to zymography. Cell lysates were subjected to western blot analysis by the indicated antibodies and actin expression was used as a reference. (D) Cells were treated with FAK siRNA, PP2, or both, stimulated with IL-1 $\beta$ and subjected to an invasion assay. Data represent the mean \pm SD from three independent experiments.

IL-1b-induced cell invasion was suppressed by PP2 treatment, and the combined treatment of PP2 and FAK siRNA significantly reduced cell invasion ( $\mathrm{P}=0.0014$; Fig. 3D). Therefore, FAK and Src are both required for IL-1b-mediated MMP-9 production and cell invasion.

$I L-1 b$-mediated MMP-9 production is dependent on the activation of FAK. To confirm the role of FAK in IL-1b-induced MMP-9 production, FAK-Ko cells and FAK-Wt cells (generated by transfecting FAK-Ko cells with wild-type FAK) were used. The cells were serum-starved and stimulated with varying concentrations of IL-1b $(0,1,3,5$, and $10 \mathrm{nM})$ before undergoing gelatin zymography. The results demonstrated that IL-1b stimulation increased the production of MMP-9 by FAK-Wt cells (Fig. 4A). By contrast, an increase of MMP-2 production stimulated by IL-1b was limited in FAK-Wt cells. FAK-Ko cells responded weakly to IL-lb treatment, and the production of MMP-9 was markedly lower than untreated FAK-Wt cells (Fig. 4A). These results indicate that FAK is crucial for IL-1b-mediated MMP-9 production.

The effect of IL-1b stimulation in FAK-Wt cells was subsequently examined, to identify whether FAK and Src were activated. FAK-Wt cells were serum-starved, stimulated with $3 \mathrm{nM} \mathrm{IL-1b}$, and the expression of phosphorylated FAK and Src was examined by western blot analysis. This demonstrated that IL-1b enhanced the tyrosine phosphorylation of FAK and Src in FAK-Wt cells (Fig. 4B and C).

Finally, to investigate the functional role of FAK for MMP-9 production, cell lines expressing mutant FAK were established. FAK-Y397F and FAK-Y925F are mutant FAKs in which Tyr397 and Tyr925 are replaced with phenylalanine, respectively. FAK-KD lacks kinase activity due to replacement of lysine 454 with arginine. Cell lines expressing these mutants did not increase MMP-9 expression in the presence of IL-1b (Fig. 4D). These data suggest a critical role of FAK activity in IL-1b-dependent MMP-9 production.

\section{Discussion}

IL-1b is abundant at tumor sites, and it induces the expression of various genes to facilitate malignant cell invasion (7). Src and FAK are critical regulators that control cell attachment, migration and signal transduction which are associated with invasion and metastasis (27-29). The present report investigated 
A

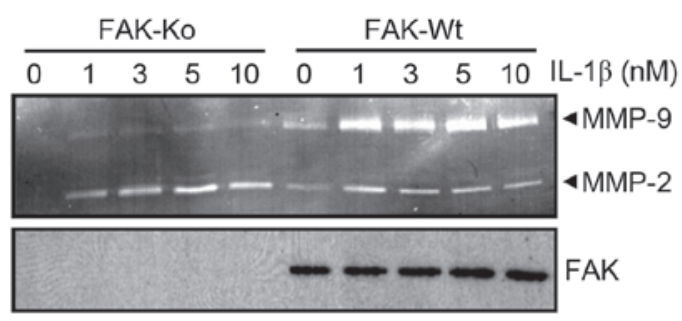

B

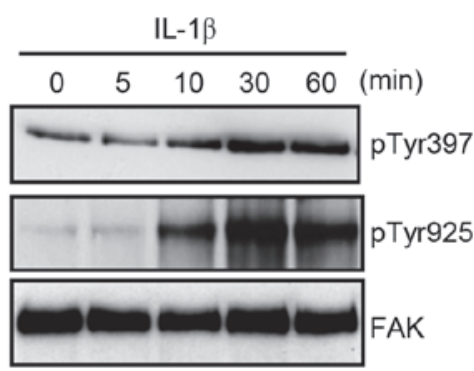

C

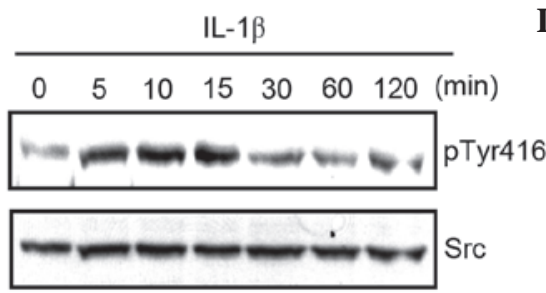

D

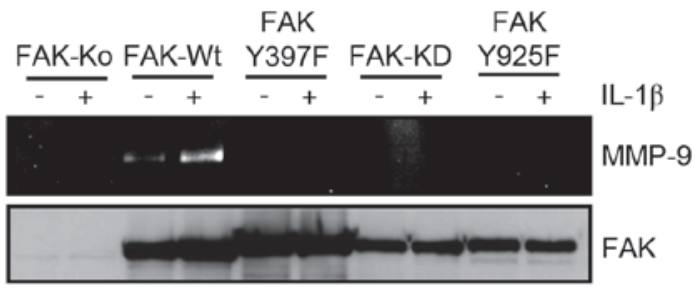

Figure 4. FAK is required for IL-1 $\beta$ induced MMP-9 production in mouse fibroblast cells. (A) FAK-Ko and FAK-Wt cells were serum-starved and stimulated with the indicated doses of IL-1 $\beta$ for $16 \mathrm{~h}$. Conditioned media were collected and subjected to gelatin zymography (upper panel). FAK expression in cell lysates is presented in the lower panel, and acted as a reference (B) FAK-Wt cells were serum-starved and stimulated with 3 nM of murine IL-1 $\beta$ for the indicated times. Tyr397 and Tyr925 phosphorylation of FAK was assessed by western blot analysis. FAK expression was used as a reference. (C) FAK-Wt cells were serum-starved and treated with $3 \mathrm{nM}$ murine IL-1 $\beta$ for the indicated times, and Tyr416 phosphorylation of Src was assessed by western blot analysis. Src expression was used as a reference. (D) Cells expressing either wild-type or mutant FAK were serum-starved and stimulated with or without 3 nM of IL-1 $\beta$ for $16 \mathrm{~h}$. Conditioned media were collected and subjected to gelatin zymography (upper panel). Expression of FAK in cell lysates was assessed by western blot analysis (lower panel). FAK expression was used as a reference.

whether Src and FAK are required for IL-1b-mediated MMP-9 production and cell invasion. Using FAK-Ko mouse fibroblasts and FAK siRNA-transfected MCF-7 cells, it was demonstrated that FAK is essential for IL-1b-induced MMP-9 production and cell invasion. Furthermore, suppression of Src activity by a chemical inhibitor decreased IL-1b-induced MMP-9 production and cell invasion, indicating that Src activation is required to induce MMP-9 via stimulation of IL-1b. These results demonstrate that activation of the Src/FAK pathway is crucial for cell invasion following IL-1b stimulation.

MMPs are major proteolytic enzymes required for tumor invasion and angiogenesis. Among MMPs, MMP-9 secretion is observed in different types of cancer, and its production is regulated by extracellular stimuli, such as growth factors and cytokines (30). Although the signaling pathways required for MMP-9 production differ depending on the extracellular stimuli, Erk activation seems to be essential $(14,31)$. In the current study, it was observed that inhibiting the Src/FAK pathway significantly reduced the activation of Erk by IL-1b. It had previously been demonstrated that phosphorylating Tyr397 and Tyr925 induces the activation of the Ras/Erk pathway (19). In the present study, cells expressing mutant FAK, which consisted of Tyr397 and Try925 substituted to alanine, exhibited only a limited increase of MMP-9 production following IL-1b stimulation. These results suggest that activation of the $\mathrm{Src} / \mathrm{FAK}$ pathway is required for the Erk activation by IL-1b, which in turn promotes MMP-9 production.

Previous studies have indicated that Src and FAK are related to cancer progression and invasion (32-34). Elevated Src expression has been observed in different types of cancer, including colon, breast, pancreatic and gastric cancer (27). In addition, genetic analysis has revealed an activating mutation in the C-terminus of Src in a subset of metastatic colon cancers (35). Overexpression of FAK has been observed in various types of invasive cancer, and FAK activity in malignant cells is correlated with invasiveness (36). Both Src and FAK are critical for the assembly of focal adhesions and cytoskeleton to induce tumor cell invasion (37). In addition to these critical functions, the current study indicated that Src and FAK are required for IL-1b-induced cell invasion and MMP-9 production. Taken together, these results indicate that the Src/ FAK pathway plays a pivotal role in inflammation-mediated tumor cell invasion.

IL-1b promotes MMP-9 production and cell invasion in non-metastatic MCF-7 breast cancer cells. Src and FAK activation are important for MMP-9 production and cell invasion by IL-1b stimulation and IL-1b-induced Erk activation is dependent on the activation of the Src/FAK pathway. Systemic treatment of mice with the IL-1 receptor antagonist (IL-1Ra), a physiological inhibitor of IL-1 signaling, inhibits tumor growth and metastasis, indicating that targeting IL-1b signaling is a promising therapy for cancer (9). Both Src and FAK have been extensively studied over the last decade. However, therapeutically targeting Src and FAK has only generated substantial interest recently (38).

In conclusion, the results of the present study suggest that the inhibition of the Src/FAK pathway is an effective treatment for inflammation-associated tumor growth and invasion.

\section{Acknowledgements}

We thank the members and staff of the Division of Cancer Biology for their technical assistance and helpful discussion and S.K. Hanks for providing FAK mutants. This work was 
supported by a grant from the Japan Society for the Promotion of Science (grant no. 15K08302).

\section{References}

1. Grivennikov SI, Greten FR and Karin M: Immunity, inflammation, and cancer. Cell 140: 883-899, 2010.

2. Hussain SP and Harris CC: Inflammation and cancer: An ancient link with novel potentials. Int J Cancer 121: 2373-2380, 2007.

3. Hugo HJ, Saunders C, Ramsay RG and Thompson EW: New insights on COX-2 in chronic inflammation driving breast cancer growth and metastasis. J Mammary Gland Biol Neoplasia 20: $109-119,2015$.

4. Harris RE, Casto BC and Harris ZM: Cyclooxygenase-2 and the inflammogenesis of breast cancer. World J Clin Oncol 5: 677-692, 2014

5. Hanahan D and Weinberg RA: Hallmarks of cancer: The next generation. Cell 144: 646-674, 2011.

6. Lu H, Ouyang W and Huang C: Inflammation, a key event in cancer development. Mol Cancer Res 4: 221-233, 2006.

7. Apte RN, Dotan S, Elkabets M, White MR, Reich E, Carmi Y, Song X, Dvozkin T, Krelin Y and Voronov E: The involvement of IL-1 in tumorigenesis, tumor invasiveness, metastasis and tumor-host interactions. Cancer Metastasis Rev 25: 387-408, 2006.

8. Apte RN, Krelin Y, Song X, Dotan S, Recih E, Elkabets M, Carmi Y, Dvorkin T, White RM, Gayvoronsky L, et al: Effects of micro-environment- and malignant cell-derived interleukin-1 in carcinogenesis, tumour invasiveness and tumour-host interactions. Eur J Cancer 42: 751-759, 2006.

9. Voronov E, Shouval DS, Krelin Y, Cagnano E, Benharroch D, Iwakura Y, Dinarello CA and Apte RN: IL-1 is required for tumor invasiveness and angiogenesis. Proc Natl Acad Sci USA 100 2645-2650, 2003

10. Voronov E, Carmi Y and Apte RN: Role of IL-1-mediated inflammation in tumor angiogenesis. Adv Exp Med Biol 601: 265-270, 2007.

11. Tu S, Bhagat G, Cui G, Takaishi S, Kurt-Jones EA, Rickman B, Betz KS, Penz-Oesterreicher M, Bjorkdahl O, Fox JG and Wang TC: Overexpression of interleukin-1beta induces gastric inflammation and cancer and mobilizes myeloid-derived suppressor cells in mice. Cancer Cell 14: 408-419, 2008.

12. Weber A, Wasiliew P and Kracht M: Interleukin-1 (IL-1) pathway. Sci Signal 3: $\mathrm{cm} 1,2010$

13. Yokoo T and Kitamura M: Dual regulation of IL-1 beta-mediated matrix metalloproteinase- 9 expression in mesangial cells by NF-kappa B and AP-1. Am J Physiol 270: F123-F130, 1996.

14. Ruhul Amin AR, Senga T, Oo ML, Thant AA and Hamaguchi M: Secretion of matrix metalloproteinase- 9 by the proinflammatory cytokine, IL-1beta: A role for the dual signalling pathways, Akt and Erk. Genes Cells 8: 515-523, 2003.

15. Bauvois B: New factors of matrix metalloproteinases MMP-2 and MMP-9 as cell surface transducers: Outside-in signaling and relationship to tumor progression. Biochim Biophys Acta 1825: 29-36, 2012.

16. Ren F, Tang R, Zhang X, Madushi WM, Luo D, Dang Y, Li Z, Wei $\mathrm{K}$ and Chen G: Overexpression of MMP family members function as prognostic biomarker for breast cancer patients: A systemic review and meta-analysis. PLoS One 10: e0135544, 2015.

17. Playford MP and Schaller MD: The interplay between Src and integrins in normal and tumor biology. Oncogene 23: 7928-7946, 2004.

18. Roskoski R Jr: Src kinase regulation by phosphorylation and dephosphorylation. Biochem Biophys Res Commun 331: 1-14, 2005.
19. Mitra SK and Schlaepfer DD: Integrin-regulated FAK-Src signaling in normal and cancer cells. Curr Opin Cell Biol 18: 516-523, 2006.

20. Hall JE, Fu W and Schaller MD: Focal adhesion kinase: Exploring FAK structure to gain insight into function. Int Rev Cell Mol Biol 228: 185-225, 2011.

21. Calalb MB, Polte TR and Hanks SK: Tyrosine phosphorylation of focal adhesion kinase at sites in the catalytic domain regulates kinase activity: A role for Src family kinases. Mol Cell Biol 15 954-963, 1995.

22. Schlaepfer DD and Hunter T: Evidence for in vivo phosphorylation of the Grb2 SH2-domain binding site on focal adhesion kinase by Src-family protein-tyrosine kinases. Mol Cell Biol 16: 5623-5633, 1996

23. Rahman MA, Senga T, Ito S, Hyodo T, Hasegawa $H$ and Hamaguchi M: S-nitrosylation at Cystein 498 of c-Src tyrosine kinase regulates nitoric oxide-mediated cell invasion. J Biol Chem 285: 3806-3814, 2010.

24. Ilić D, Furuta Y, Kanazawa S, Takeda N, Sobue K, Nakatsuji N, Nomura S, Fujimoto J, Okada M and Yamamoto T: Reduced cell motility and enhanced focal adhesion contact formation in cells from FAK-deficient mice. Nature 377: 539-544, 1995.

25. Mon NN, Hasegawa H, Thant AA, Huang P, Tanimura Y, Senga T and Hamaguchi M: A role for focal adhesion kinase signaling in tumor necrosis factor-alpha-dependent matrix metalloproteinase-9 production in a cholangiocarcinoma cell line, CCKS1. Cancer Res 66: 6778-6784, 2006.

26. Shibata K, Kikkawa F, Nawa A, Thant AA, Naruse K, Mizutani S and Hamaguchi M: Both focal adhesion kinase and c-Ras are required for the enhanced matrix metalloproteinase 9 secretion by fibronectin in ovarian cancer cells. Cancer Res 58: 900-903, 1998.

27. Rajshankar D, Downey GP and McCulloch CA: IL-1 $\beta$ enhances cell adhesion to degraded fibronectin. FASEB J 26: 4429-4444, 2012.

28. Frame MC: Src in cancer: Deregulation and consequences for cell behavior. Biochim Biophy Acta 1602: 114-130, 2002.

29. Avizienyte E and Frame MC: Src and FAK signalling controls adhesion fate and the epithelial-to-mesenchymal transition. Curr Opin Cell Biol 17: 542-547, 2005

30. Kessenbrock K, Plaks V and Werb Z: Matrix metalloproteinases: Regulators of the tumor microenvironment. Cell 141: 52-67, 2010.

31. Sen T, Dutta A, Maity G and Chatterjee A: Fibronectin induces matrix metalloproteinase-9 (MMP-9) in human laryngeal carcinoma cells by involving multiple signaling pathways. Biochimie 92: 1422-1434, 2010.

32. Siesser PM and Hanks SK: The signaling and biological implications of FAK overexpression in cancer. Clin Cancer Res 12: 3233-3237, 2006

33. Summy JM and Gallick GE: Src family kinases in tumor progression and metastasis. Cancer Metastasis Rev 22: 337-358, 2003.

34. Mon NN, Ito S, Senga T and Hamaguchi M: FAK signaling in neoplastic disorders: A linkage between inflammation and cancer. Ann NY Acad Sci 1086: 199-212, 2006.

35. Irby RB, Mao W, Coppola D, Kang J, Loubeau JM, Trudeau W, Karl R, Fujita DJ, Jove R and Yeatman TJ: Activating SRC mutation in a subset of advanced human colon cancers. Nature Genet 21: 187-190, 1999.

36. McLean GW, Carragher NO, Avizienyte E, Evans J, Brunton VG and Frame MC: The role of focal-adhesion kinase in cancer - a new therapeutic opportunity. Nat Rev Cancer 5: 505-515, 2005.

37. Brunton VG and Frame MC: Src and focal adhesion kinase as therapeutic targets in cancer. Curr Opin Pharmacol 8: 427-432, 2008.

38. Yoon H, Dehart JP, Murphy JM and Lim ST: Understanding the role of FAK in cancer: Inhibitors, genetic models, and new insights. J Histochem Cytochem 62: 114-128, 2015. 